\title{
Nuevas técnicas en el estudio del asma
}

\section{New techniques in the study of asthma}

\section{J.M. Olaguíbel, M.J. Álvarez, P. Uribe, B.E. García, A.I. Tabar}

\section{RESUMEN}

El asma es consecuencia de la inflamación crónica de la vía aérea que se manifiesta funcionalmente como obstrucción variable al flujo aéreo e hiperreactividad obstruccion variable al flujo aereo hiperreactividad bronquial. Con relativa frecuencia no disponemos de indices objetivos que nos permitan diagnosticar y controlar la enfermedad. Este artículo revisa técnicas adicionales de evaluación del funcionalismo pulmonar (provocación bronquial con adenosín-monofosfato, cuantificación de la resistencia al flujo aéreo por oscilometría de impulsos o pletismografía corporal) y de la respuesta inmunoinflamatoria bronquial (inducción de esputo, condensación de aire exhalado, monitorización de óxido nítrico en aire exhalado) y nuestra experiencia con su utilización.

Palabras clave. Asma. Inflamación. Adenosínmonofosfato. Oscilometría de impulsos. Pletismografía corporal Esputo inducido Oxido nítrico. Condensados de aire exhalado.

\section{ABSTRACT}

Asthma is a chronic inflammatory disease of the airways that is functionally characterized by variability of lung function and bronchial hyper-responsiveness Its diagnosis and management is commonly hampered by the lack of objective parameters. This article reviews additional techniques for evaluating both, lung function (bronchial challenge with adenosinmonophosphate, lung resistance measurement by either the impulse oscillometry system or whole body either the impulse oscillometry system or whole body
pletismography) and bronchial immuno-inflammatory
response (induced sputum, condensates of exhaled air, response (induced sputum, condensates of exhaled air
monitoring of nitric oxide in exhaled air), as well as our experience with them

Key words. Asthma. Inflammation. Adenosinmonophosphate. Impulse oscillometry system. Whole body pletismography. Induced sputum. Nitric oxide. Condensates of exhaled air
Sección de Alergología. Hospital Virgen del Camino. Pamplona

\section{Correspondencia:}

J.M. Olaguíbel Rivera

Servicio de Alergología

C.S. Conde Oliveto

Plaza de la Paz s/n.

31002 Pamplona

Tfno. 948429303

jmolaguir@cfnavarra.es 


\section{INTRODUCCIÓN}

Clínicamente el asma se caracteriza por crisis de obstrucción al flujo aéreo bronquial, más o menos reversibles, de forma espontánea o tras la administración de broncodilatadores $^{1}$. Con frecuencia, la percepción de los síntomas de obstrucción bronquial es subestimada tanto por el paciente como por el médico ${ }^{3} \mathrm{y}$, dado el carácter episódico del asma, en numerosas ocasiones no es posible objetivar en la maniobra de espirometría forzada el patrón obstructivo reversible tras la inhalación de broncodilatadores propio del asma. Tampoco se debe olvidar que un considerable número de pacientes, entre los que destacan niños menores de 6 años, no es capaz de realizar maniobras espirométricas reproducibles ${ }^{4}$.

Estudios de lavado broncoalveolar han constatado que la base anatómica del asma es, incluso en los casos más leves o durante los periodos asintomáticos, la inflamación crónica eosinofílica de la vía aérea ${ }^{5}$. Por ello, los fármacos anti-inflamatorios, en especial los corticoides inhalados, se han impuesto como el pilar básico de su tratamiento. Se ha constatado el beneficio del inicio precoz del tratamiento antiinflamatorio continuado incluso en el asma leve y de corta evolución ${ }^{6}$; sin embargo, algunos autores han comunicado que estos fármacos no están completamente exentos de efectos secundarios sistémicos ${ }^{7}$.

Como consecuencia de lo anteriormente expuesto, se impone la necesidad de disponer de índices que nos permitan realizar un diagnóstico y seguimiento más preciso del asma bronquial, evitando por una parte, el retraso en el establecimiento del tratamiento adecuado y por otra, la administración de fármacos innecesarios. A continuación se expondrán brevemente algunas de las técnicas contrastadas de evaluación funcional y de inflamación de la vía aérea, recientemente desarrolladas, así como nuestra experiencia en su utilización.

\section{TÉCNICAS DE ESTUDIO DEL FUNCIONALISMO PULMONAR}

\section{Pruebas de provocación bronquial}

La hiperreactividad bronquial (HRB), definida como la sensibilidad exagerada de la vía aérea a diversos tipos de estímulos: físicos (ejercicio, frío) o farmacológicos (metacolina, histamina), se puede evidenciar en nuestro laboratorio mediante las pruebas de provocación bronquial (PB). Estas técnicas se basan en que en individuos susceptibles, la aplicación controlada de estímulos broncoconstrictores entre los que destacan la histamina y la metacolina ya que son los más estandarizados, induce grados objetivos de obstrucción al flujo aéreo. Los resultados se expresan como la dosis de agonista necesaria para reducir el valor de $\mathrm{FEV}_{1}$ hasta un valor prefijado respecto a sus valores basales, generalmente el $20 \%$ (índice $\mathrm{PD}_{20}$ ). La sensibilidad de ambos agonistas es muy alta, pero no así su especificidad ya que se pueden detectar respuestas positivas en pacientes con enfermedades respiratorias diferentes al asma, en sujetos con rinitis o incluso en individuos sanos ${ }^{8}$. Por ello, la aplicación clínica principal de la PB con metacolina o histamina se basa en su elevado valor predictivo negativo: excepto en situaciones puntuales como es el caso de individuos con asma alérgica en los que ha cesado totalmente la exposición ambiental, la falta de detección de un valor de $\mathrm{PD}_{20}$ descarta el diagnóstico de asma ${ }^{8}$. No obstante, debido a su baja especificidad, la detección de valores de $\mathrm{PD}_{20}$ no es sinónimo de asma ni indicador de su severidad.

El examen de las curvas completas dosis-respuesta con metacolina permite evaluar la existencia de una meseta de máxima respuesta bronquial (MRB), definida como la máxima contracción que experimenta el músculo liso ante la administración del agonista, de modo que sucesivas dosis de éste no inducen mayor obstrucción al flujo aéreo. Como se ha comentado anteriormente, en ocasiones se observan valores de $\mathrm{PD}_{20}$ en sujetos no asmáticos en los que sin embargo, la inhalación de dosis sucesivas de agonista suele inducir, probablemente como consecuencia de la puesta en funcionamiento de mecanismos protectores, un grado máximo de deterioro de la función pulmonar. Con frecuencia, para objetivar la meseta de MRB es preciso inducir importantes descensos de valor de $\mathrm{FEV}_{1}$, incluso del $60 \%$ respecto a su valor basal. La MRB es presumiblemente la prin- 
cipal anormalidad fisiopatológica del asma ya que su detección o más aún, su ausencia a altos grados de obstrucción bronquial supone para el paciente un importante riesgo de presentar crisis severas de asma sin que se activen los mecanismos de protección de las vías aéreas, pudiendo incluso derivar en fallo respiratorio ${ }^{9}$. La objetivación de una meseta de MRB permite una mejor separación entre asmáticos y sujetos con rinitis alérgica ${ }^{10}$ y el valor de la pendiente de la curva dosis-respuesta es el índice que mejor discrimina sujetos con rinitis y asma leve respecto a los que exclusivamente presentan síntomas nasales $^{11}$. Paralelamente, en pacientes con asma leve, el grado de obstrucción necesario para obtener un "plateau" de MRB se correlaciona directamente con la eosinofilia en esputo ${ }^{12}$

Estos datos sugieren que el estudio de curvas completas dosis-respuesta con metacolina desempeñaría un papel en el diagnóstico precoz del asma. Sin embargo, dado el alto grado de obstrucción de la vía aérea que es necesario inducir, es recomendable que esta prueba se realice en centros especializados y con experiencia. Adicionalmente la realización de esta técnica es prolongada lo que limita su utilidad en la práctica clínica. Recientemente se han realizado estudios de provocación con otros agentes como el adenosín monofosfato (AMP) que, a través de la activación de los mastocitos de la vía aérea y la consiguiente liberación de mediadores preformados causa de forma indirecta bronconstricción. Mientras que la sensibilidad bronquial a la metacolina depende de la inflamación bronquial y el calibre basal de la vía aérea $\left(\mathrm{FEV}_{1}\right)$, la sensibilidad a AMP depende fundamentalmente del grado de inflamación de la mucosa bronquial ${ }^{13}$. Esta teoría se basa en la observación de que el tratamiento antiinflamatorio reduce de forma más sustancial la sensibilidad bronquial al AMP que a la metacolina que también depende del incremento en los valores de $\mathrm{FEV}_{1}{ }^{14-16}$. La PB con AMP es reproducible $^{17}$ y a pesar de que muestra menor sensibilidad que la realizada con metacoli$\mathrm{na}^{18}$, proporciona una mayor especificidad para el asma ${ }^{13}$, especialmente en el caso del asma alérgico ${ }^{19}$. Sin embargo, un núme- ro de sujetos con rinitis alérgica exhibe sensibilidad bronquial al AMP ${ }^{20}$, correlacionándose sus valores de $\mathrm{PD}_{20}$ con la eosinofilia en esputo ${ }^{21}$. El hecho de que éstos no sean los mismos pacientes que responden a metacolina, sugiere que los mecanismos por los que actúan ambas sustancias son diferentes.

\section{Cuantificación de resistencias bronquiales}

El índice utilizado para cuantificar la respuesta bronquial en las provocaciones bronquiales es el volumen espiratorio forzado en el primer segundo $\left(\mathrm{FEV}_{1}\right)^{8}$. Ciertos grupos de pacientes, en especial niños menores de 6 años, no son capaces de realizar maniobras reproducibles de espirometría forzada. De ahí la necesidad de disponer de índices válidos que permitan evaluar cambios en el calibre de la vía aérea.

La evaluación de la resistencia al flujo aéreo mediante la pletismografía corporal, basada en la relación entre la presión y el volumen en condiciones isotermas y bajo volumen constante, ha sido ampliamente constatada en la literatura ${ }^{22}$. Esta técnica sólo requiere respiración a volumen corriente por lo que puede ser realizada por la mayor parte de los individuos. Sin embargo, precisa un equipamiento sofisticado y por tanto, no siempre disponible. Hay que apuntar además el rechazo de algunos sujetos a permanecer en la cabina cerrada $^{23}$.

El sistema de oscilometría de impulsos (IOS) se basa en la relación entre pulsos de presión aplicados al sistema respiratorio (a través de un altavoz adaptado a la boquilla) y la respuesta resultante de la vía aérea. Los índices con ella obtenidos reflejan la resistencia, en especial de las vías aéreas centrales y la elasticidad pulmonar. Esta técnica es sencilla ya que requiere exclusivamente ciclos de respiración de 20-30 segundos de duración a volumen corriente. Estas características permiten que pueda ser utilizada en la evaluación de la función pulmonar en sujetos que no colaboran en las maniobras forzadas de espirometría, como niños de más de dos años de edad. Se ha constatado que los índices obtenidos con esta técnica son altamente reproducibles y 
sensibles para constatar los cambios originados en el calibre bronquial por la administración de broncodilatadores ${ }^{24}$, o metacolina $^{25}$. Recientemente un estudio piloto realizado en nuestra sección en un grupo de niños asmáticos de edades comprendidas entre los 3 y 6 años mostró una reproducibilidad basal de las mediciones de IOS inferior al 5\%. La inhalación de salbutamol indujo cambios en las resistencias cuantificadas por IOS paralelos a los observados en sRaw (pletismografia corporal) y $\mathrm{FEV}_{1}$ (espirometría forzada), hallándose un grado aceptable de correlación entre las tres técnicas. Por último, cabe destacar la ventaja adicional que supone que el equipamiento de la oscilometría de impulsos sea portátil, lo que permite su aplicación en diferentes ámbitos.

\section{TÉCNICAS DE ESTUDIO DE LA INFLAMACIÓN BRONQUIAL}

Durante las últimas décadas, los fármacos antiinflamatorios se han convertido en el pilar del tratamiento del asma. Por lo tanto, parece lógico disponer de índices que nos permitan cuantificar el grado de inflamación bronquial para optimizar el tratamiento antiasmático. El carácter invasivo de las técnicas clásicas de estudio de la mucosa bronquial: biopsia, cepillado, lavado broncoalveolar, limita su aplicación en el diagnóstico y monitorización de una enfermedad tan prevalente como el asma bronquial. Por otra parte, los marcadores de inflamación eosinofílica (eosinófilos, proteínas básicas) se incrementan en muestras de sangre o de orina de pacientes asmáticos, pero también en las de sujetos con dermatitis atópica o rinitis alérgi$\mathrm{ca}^{26}$, lo que limita su utilidad en el asma.

La técnica de esputo inducido, basada en la inhalación de una solución hipertónica que aumenta el volumen de las secreciones bronquiales, permite obtener de forma segura y no invasiva material representativo de vías aéreas inferiores. El tratamiento de la muestra con agentes dispersantes de la matriz de moco (p. ej. ditiotreitol) aumenta el rendimiento de la identificación celular y permite obtener una fase líquida en la que cuantificar mediadores solubles de la inflamación.
Durante la última década, se ha demostrado que los índices de inflamación eosinofílica (porcentaje de eosinófilos y valores de proteína catiónica del eosinófilo) obtenidos con esta técnica son reproducibles ${ }^{27,28}$ y muestran valores más elevados en el asma que en otras enfermedades respiratorias como la bronquitis crónica obstructiva $^{29}$ o la rinitis alérgica ${ }^{26}$. Los indicadores de inflamación eosinofílica se modifican de forma paralela a la actividad de la enfermedad, incrementándose tras la exposición al alergeno en individuos susceptibles $^{30}$ y reduciéndose tras la administración de tratamiento antiinflamatorio ${ }^{31}$.

Sin embargo, la técnica de inducción de esputo requiere colaboración por parte del paciente, de modo que en un porcentaje de sujetos, en especial niños, no es posible obtener una muestra adecuada ${ }^{32}$. El hecho de que sea una técnica que consume un tiempo relativamente prolongado y que la obtención de resultados sea diferida, plantea inconvenientes en su aplicación en la práctica clínica. El óxido nítrico (NO) es un pequeño metabolito liberado en el paso de L-argirina a L-citrulina que ejerce un efecto dual sobre la vía aérea. A bajas concentraciones debido a sus propiedades como broncodilatador, vasodilatador y antimicrobiano ejerce efectos beneficiosos. Sin embargo, cuando se libera a altas concentraciones, como consecuencia de estímulos inflamatorios, resulta dañino para la vía aérea ya que incrementa el edema de la mucosa y favorece la síntesis de radicales libres, potentes oxidantes con actividad citotóxica e implicados en la descamación epitelial. La cuantificación de los niveles de óxido nítrico en aire exhalado mediante quimioluminiscencia es una técnica de sencilla realización que aporta resultados inmediatos y reproducibles ${ }^{33}$. Los valores de NO en aire exhalado son superiores en asmáticos que en individuos $\operatorname{sanos}^{34}$ y descienden tras la administración de tratamiento con corticoides inha$\operatorname{lados}^{35} \mathrm{o}$ antileucotrienos ${ }^{36}$. A la inversa, se ha constatado elevación de los valores de NO al deteriorarse la situación clínica en el contexto de la reducción del tratamiento antiinflamatorio $^{37}$ o del incremento de la exposición alergénica en individuos susceptibles $^{38}$. De acuerdo con estos datos, es 
posible inferir la existencia de una estrecha asociación entre los niveles de $\mathrm{NO}$ en aire exhalado y el grado de activación inmunoinflamatoria en el asma lo que apoya la utilidad de la monitorización de este metabolito en el control del asma. Estas perspectivas se ven sin embargo reducidas por la falta de especificidad del $\mathrm{NO}$, ya que sus valores se incrementan también en otras entidades que cursan con inflamación del tracto bronquial como por ejemplo, la bronquitis crónica obstructiva, la bronquiolitis obstructiva, el síndrome de distrés respiratorio del adulto $\mathrm{o}$ incluso, en la rinitis alérgica y en el tabaquismo ${ }^{39}$. Por otra parte, las mediciones de NO varían considerablemente en función de factores técnicos, fisiológicos y ambientales $^{39}$ por lo que resulta obligado conseguir una mayor estandarización de la técnica con el establecimiento de los valores y rangos de variación normal que permita una mayor difusión en la práctica clínica.

El flujo de aire respirado origina turbulencias en los conductos bronquiales que aerosolizan el líquido que baña la pared bronquial. Basado en este principio, la condensación del aire exhalado por el paciente, mediante un sistema eléctrico refrigerante nos permite obtener una fase líquida en la que cuantificar mediadores volátiles de la inflamación ${ }^{40,41}$. Esta técnica no invasiva sólo requiere la respiración a volumen corriente durante un periodo de tiempo que oscila entre 5 y 15 minutos. Al igual que ocurre con el NO, la acidosis leve de la vía aérea es beneficiosa ya que frena el crecimiento de microorganismos. En cambio, la acidificación intensa favorece la inflamación eosinofílica con la liberación en cascada de mediadores, aumenta la viscosidad del moco y resulta tóxica para las células endoteliales. La determinación del $\mathrm{pH}$ en las muestras de aire exhalado es sencilla y permite obtener resultados inmediatos. Diversos estudios han demostrado que las mediciones individuales de $\mathrm{pH}$ en asmáticos son reproducibles ${ }^{42}$ y se relacionan estrechamente con el grado de inflamación eosinofílica ${ }^{42}$ pero no con el calibre bronquial ${ }^{41}$. En cambio, en la bronquitis crónica obstructiva, la acidificación de la vía aérea se correlaciona con el infiltrado de neutrófilos ${ }^{42}$. Debido a esta falta de especificidad, diversos estudios han evaluado la posibilidad de cuantificar en las muestras de condensado de aire exhalado otros marcadores que permitan mayor aproximación a la enfermedad asmática. Para evitar el posible efecto dilucional de la saliva, los resultados se han expresado como cocientes, de modo que el cociente entre interleucina-4/interferon gamma es un indicador de inflamación eosinofílica que se encuentra elevado en el asma $^{43}$. Paralelamente, se ha descrito en asmáticos una mayor actividad oxidativa que se manifiesta por el incremento de los cocientes entre peróxido de hidrógeno y 8 isoprostano y entre aldehídos y glutation, así como del metabolismo del óxido nítrico a través del aumento de la relación entre nitratos y nitritos ${ }^{42,44}$

\section{CONCLUSIÓN}

Resulta imperativa la necesidad de disponer de índices objetivos que nos permitan diagnosticar y ajustar el tratamiento del paciente asmático. En los últimos años, se han desarrollado diversas técnicas en este aspecto, cada una de ellas con ventajas y limitaciones. De acuerdo a nuestra experiencia, contrastada con la de otros autores, cada una de ellas posee una serie de indicaciones bien definidas. Así, debido a su elevado valor predictivo negativo, las pruebas de provocación bronquial con metacolina o histamina permiten descartar el diagnóstico de asma. En cambio, la evaluación de la HRB mediante adenosínmonofosfato, más dependiente de la inflamación de la vía aérea puede ser adecuada en el estudio del asma alérgico. La cuantificación de la resistencia de la vía aérea mediante pletismografía corporal u oscilometría de impulsos, proporciona índices objetivos de función pulmonar en individuos en los que la maniobra espirométrica forzada no resulta posible. Por otra parte, la inducción de esputo o la condensación de aire exhalado nos permite obtener de forma no invasiva material bronquial en el que cuantificar la respuesta inmunoinflamatoria. Por último, la monitorización de los valores de óxido nítrico en aire exhalado es un método simple y rápido de evaluar la respuesta y la adherencia al tratamiento antiasmático. Estas técnicas apor- 
tan información sobre diferentes aspectos de la enfermedad asmática y su utilización en la práctica clínica debería contribuir a obtener información adicional que permita optimizar el diagnóstico y tratamiento de una patología tan frecuente como es el asma bronquial.

\section{BIBLIOGRAFÍA}

1. Guidelines for the diagnosis and management of asthma. National Heart, Lung, and Blood Institute. National Asthma Education Program. Expert Panel Report. J Allergy Clin Immunol 1991; 88: 425-534.

2. Salome CM, Xuan W, Gray EJ, Belooussova E, PEAT JK. Perception of airway narrowing in a general population sample. Eur Respir J 1997; 10: 1052-1058.

3. Shim C, WiLliams M. Evaluation of the severity of asthma: patient versus phisicians. Am J Med 1980; 68: 11-13.

4. Standardization of Spirometry, 1994 Update. American Thoracic Society. Am J Respir Crit Care Med 1995; 152: 1107-1136.

5. DJUKANOVIC R, LAI CK, WILSON JW. Bronchial mucosal manifestations of atopy: a comparison of markers of inflammation between atopic asthmatics, atopic nonasthmatics and healthy controls. Eur Respir J 1992; 5: 538-544.

6. HAAHTELA T. The long-term influence of therapeutic interventions in asthma with emphasis on inhaled steroids and early disease. Clin Exp Allergy 1998; 5: 133-140.

7. GOLDBERG S, EiNOT T, AlguR N. Adrenal suppression in asthmatic children receiving low-dose inhaled budesonide: comparison between dry powder inhaler and pressurized metered-dose inhaler attached to a spacer. Ann Allergy Asthma Immunol 2002; 89: 566-571.

8. STERK PJ, FABBri LM, QuANJER PH. Airway responsiveness. Standardized challenge testing with pharmacological, physical and sensitizing stimuli in adults. Report Working Party Standardization of Lung Function Tests, European Community for Steel and Coal. Official Statement of the European Respiratory Society. Eur Respir J Suppl 1993; 16: $53-83$

9. STERK PJ, BEL EH. Bronchial hyperresponsiveness: the need for a distinction between hypersensitivity and excessive airway narrowing. Eur Respir J 1989; 2: 267274.

10. Prieto L, Gutierrez V, Morales C, Marin J. Differences in sensitivity, maximal response and position of the concentration-response curve to methacholine between asthmatics, patients with allergic rhinitis and healthy subjects. Respir Med 1998; 92: 88-94.

11. Alvarez-Puebla M, García-Figueroa B, TabarPurroy A, Olaguibel-Rivera J. Discriminant analysis in allergic rhinitis and asthma: methacholine dose-response slope allows a good differentiation between mild asthma and rhinitis. Resp Med 2002.

12. Álvarez-Puebla MJ, Castillo R, Rey A, ORtega N, Blanco C, Carrillo T. Sputum eosinophilia and maximal airway narrowing in Dermatophagoides pteronyssinus allergic rhinitis patients. Only rhinitis or rhinitis plus mild asthma? Chest 2002; 122: 1560-1565.

13. De Meer G, HeEderiK D, Postma DS. Bronchial responsiveness to adenosine 5'. monophosphate (AMP) and methacholine differ in their relationship with airway allergy and baseline FEV(1). Am J Respir Crit Care Med 2002; 165: 327-331.

14. van den Berge M, Kerstjens HA, Meijer RJ. Corticosteroid-induced improvement in the PC20 of adenosine monophosphate is more closely associated with reduction in airway inflammation than improvement in the PC20 of methacholine. Am J Respir Crit Care Med 2001; 164: 1127-1132.

15. VAN Schoor J, Joos GF, Pauwels RA. Indirect bronchial hyperresponsiveness in asthma: mechanisms, pharmacology and implications for clinical research. Eur Respir J 2000; 16:514-533.

16. Holgate ST, Arshad H, Stryszak P, Harrison JE Mometasone furoate antagonizes AMP induced bronchoconstriction in patients with mild asthma. J Allergy Clin Immunol 2000; 105: 906-911.

17. Egbagbe E, Pavord ID, Wilding P, THOMPSONCoOn J, TATTERSFIELD AE. Adenosine monophosphate and histamine induced bronchoconstriction: repeatability and protection by terbutaline. Thorax 1997; 52 . 239-243.

18. Fowler SJ, Dempsey OJ, Sims EJ, Lipworth BJ. Screening for bronchial hyperresponsiveness using methacholine and adenosine monophosphate. Relationship to asthma severity and beta (2)-receptor genotype. Am J Respir Crit Care Med 2000; 162: 1318-1322.

19. LudVIKSDOTTIR D, JANSON C BJORNSSON E Different airway responsiveness profiles in atopic asthma, nonatopic asthma, and Sjogren's syndrome. BHR Study Group. Bronchial hyperresponsiveness. Allergy 2000; 55: 259-265. 
20. Prieto L, Gutierrez V, Linana J, Marin J. Bronchoconstriction induced by inhaled adenosine 5'-monophosphate in subjects with allergic rhinitis. Eur Respir J 2001; 17: 64-70.

21. Polosa R, Ciamarra I, Mangano G. Bronchial hyperresponsiveness and airway inflammation markers in nonasthmatics with allergic rhinitis. Eur Respir J 2000; 15: 30-35.

22. Blonshine S. Pediatric pulmonary function testing. Resp Care Clin N Am 2000; 6: 27-40.

23. SLY P, Robertson C. A review of pulmonary function testing in children. J Asthma 1990; 27: 137-147.

24. HellinckX J, De Boeck K, Bande-Knops J, van DER Poel M, DEMEDTs M. Bronchodilator response in 3-6.5 years old healthy and stable asthmatic children. Eur Respir J 1998; 12 . 438-443.

25. Kohlhaufl M, Brand P, Scheuch G, Schulz H, HAUSSINGER K, HEYDER J. Impulse oscillometry in healthy nonsmokers and asymptomatic smokers: effects of bronchial challenge with methacholine. J Aerosol Med 2001; 14: 1-12.

26. Álvarez MJ, OlaguÍbel JM, Garcia BE, Rodriquez A, TABAR AI, URBIola E. Airway inflammation in asthma and perennial allergic rhinitis. Relationship with nonspecific bronchial responsiveness and maximal airway narrowing. Allergy 2000; 55: 355-362.

27. FAHY J, LiU J, WONG H, BOUSHLEY H. Cellular and biochemical analysis of induced sputum from asthmatic and from healthy subjects. Am Rev Respir Dis 1993; 147: 1126-1131.

28. Pizzichini E, Pizzichini M, EFthimiadis A. Indices of airway inflammation in induced sputum: reproductibility and validity of cell fluid measurements. Am J Respir Crit Care Dis 1996; 154: 308-317.

29. Virchow J, Kroegel C, Hage U, Kortsik C MATTHYS H, Werner P. Comparison of sputum ECP levels in bronchial asthma and chronic bronchitis. Allergy 1993; 48: 112-118.

30. Álvarez-Puebla M, Olaguíbel-Rivera J, URBiolaMarcilla E, Garcia B, TABar-Purroy A Determinants of allergen-induced late bronchial responses in mild asthma. Chest 2001; 119: 120-127.

31. Claman D, Boushley H, LuU J, Wong H, Fahy J. Analysis of induced sputum to examine the effects of prednisone in airway inflammation in asthmatic subjects. J Allergy Clin Immunol 1994; 94: 861-869.

32. Wilson NM, Bridge P, Spanevello A, Silverman M. Induced sputum in children: feasibility, repeatability, and relation of findings to asthma severity. Thorax 2000; 55: 768-774.
33. KHARITONOV SA, BARNES PJ. Nasal contribution to exhaled nitric oxide during exhalation against resistance or during breath holding Thorax 1997; 52:540-544.

34. Alving K, WeITZBERg E, LUNDBERg JM. Increased amount of nitric oxide in exhaled air of asthmatics. Eur Respir J 1993; 6: 1368-1370.

35. Kharitonov SA, Yates DH, Barnes PJ. Inhaled glucocorticoids decrease nitric oxide in exhaled air of asthmatic patients. Am J Respir Crit Care Med 1996; 153: 454-457.

36. Bratton DL, Lanz MJ, Miyazawa N, White CW SILKOFF PE. Exhaled nitric oxide before and after montelukast sodium therapy in schoolage children with chronic asthma: a preliminary study. Pediatr Pulmonol 1999; 28: 402-407.

37. van Rensen EL, Straathof KC, Veselic-Charvat MA, Zwinderman AH, Bel EH, Sterk PJ. Effect of inhaled steroids on airway hyperresponsiveness, sputum eosinophils, and exhaled nitric oxide levels in patients with asthma. Thorax 1999. 54: 403-408.

38. Kharitonov SA, O'Connor BJ, Evans DJ, Barnes PJ. Allergen-induced late asthmatic reactions are associated with elevation of exhaled nitric oxide. Am J Respir Crit Care Med 1995; 151: 1894-1899.

39. Ashutosh K. Nitric oxide and asthma: review. Curr Opin Pulm Med 2000; 6: 21-25.

40. Hunt JF, FAnG K, MaLIK R. Endogenous airway acidification. Implications for asthma pathophysiology. Am J Respir Crit Care Med 2000; 161: 694-699.

41. HunT J. Exhaled breath condensate: an evolving tool for noninvasive evaluation of lung disease. J Allergy Clin Immunol 2002; 110: $28-34$.

42. Kostikas $\mathrm{K}$, Papatheodorou $\mathrm{G}$, Ganas $\mathrm{K}$ Psathakis $\mathrm{K}$, Panagou $\mathrm{P}$, Loukides $\mathrm{S}$. $\mathrm{PH}$ in expired breath condensate of patients with inflammatory airway diseases. Am J Respir Crit Care Med 2002; 165: 1364-1370.

43. SHahid SK, Kharitonov SA, Wilson NM, Bush A BARNES PJ. Increased interleukin-4 and decreased interferon-gamma in exhaled breath condensate of children with asthma. Am J Respir Crit Care Med 2002; 165: 1290 1293.

44. CorRadi M, Folesani G, ANDREOLI R. Aldehydes and glutathione in exhaled breath condensate of children with asthma exacerbation. Am J Respir Crit Care Med 2003; 167: 395-399. 